\title{
The relationship between communication competence and exercise participation type: focusing on joining clubs and using fitness applications
}

\author{
Yumi Yi', Jin-Hee Seo ${ }^{2 * *}$ \\ 'DaVinch College of General Education, Chung-Ang University, Seoul, Korea \\ 2Department of Adaptive Physical Education, Baekseok University, Cheonan, Korea
}

The purpose of this study was to determine the effects of the differences in subfactors of communication competence on exercise participation types (clubs, applications use). Communication competence focus on the communication part of the individual's interpersonal competence. Therefore, communication competence can be predicted to be related to the type of exercise participation. Accordingly, the targets of this study were clubs, which had group characteristics, and fitness applications, which had individual characteristics. There was a significant difference as a result of the association between communication com- petence and the type of exercise participation. In terms of club membership, significant differences were found in communication competence skills according to exercise participation types in that self-disclosure of subjects who joined clubs was significant, social relaxation of subjects who intend to join a club was significant, and the immediacy of fitness applications users was significant.

Keywords: Communication competence, Exercise participation type, Sports club, Fitness applications, Interpersonal relation, Self-disclosure

\section{INTRODUCTION}

Passing the hyperconnectivity-based third industrial revolution, the current society of the fourth industrial revolution, with its superintelligence, is bringing numerous changes to human relations and forms of life. Hyperconnectivity, in fact, originated from a desire for disconnection, but another desire for connection was created, and the technological revolution accompanying superintelligence is giving us another paradigm of life. Such social phenomena are also influencing our exercise. The development of various applications (apps) in this technological revolution created a model such as home training by escaping traditional exercise forms that are affected by time and space limitations. The characteristics of hyperconnectivity allow online and offline club participation.

Such social changes, however, do not necessarily have meaning for or an effect on all individuals in the same way. That is why various research studies on the methods and effects of exercise par-

ticipation have been conducted. Examples of such research include investigations on the effects of interaction methods among exercise participants on exercise effects (Brawley et al., 1987; Kim and Lee, 2008) and, in terms of age, the effects of young adults' exercise experience on exercise adherence (Curtis et al., 1999). Regarding the use of fitness apps, research on factors influencing users' decision-making (Yuan et al., 2015), technology related to fitness apps (Conroy et al., 2014), and the influence and contribution of fitness apps (Gay and Leijdekkers, 2012) are also some examples. Among these studies, the work of Curtis et al. (1999) was the influence for this study to limit the age of subjects to those in their 20s. The subjects' characteristics are an important aspect of research on exercise participation methods. Among such characteristics, this study intended to focus on communication competence that leads to interpersonal relationships. Spitzberg and Cupach (1989) considered communication competence as the ability to effectively deal with others in the process of communication, while

${ }^{*}$ Corresponding author: Jin-Hee Seo (iD https://orcid.org/0000-0003-2766-1282 Department of Adaptive Physical Education, Baekseok University, 76 Munam-ro, Dongnam-gu, Cheonan 31065, Korea

Tel: +82-41-550-2207, Fax: +82-41-550-2109, E-mail: sjh0521@bu.ac.kr

Received: October 5, 2018 / Accepted: November 8, 2018 
Monge et al. (1981) considered it as the ability to maximize one's goals through communication. As discussed above, interpersonal communication competence has been variously defined, but ultimately, it can be considered as a method to effectively achieve one's communication goals in interpersonal relationships. Various scales have been developed to measure communication competence, and the competence is mostly explained by the total score of the scale. Because skills in the measured subareas are important competence variables for interpersonal communication, determining individual differences in each variable has analytical significance.

Accordingly, the goal of this study was to determine the relationship between communication competence and exercise participation type. To that end, the relationship between communication competence, club membership, and fitness app use identified the subfactors of communication competence that affect exercise participation type (clubs, apps).

\section{MATERIALS AND METHODS}

\section{Data and study population}

This study included a survey of college students in their 20s to determine the influence of interpersonal communication competence on exercise participation types. The reason for limiting subjects to college students in their 20s is that age can significantly influence the propensity for joining a club or using an app (the chosen participation types). Accordingly, this study attempted to determine the effects of communication, which is a personal trait, on joining a club or using an app, with a fixed age variable. Online and offline surveys were conducted from September 20 to October 5, 2018, and a total of 227 responses (137 offline and 90 online) were obtained. After excluding insincere responses, 182 valid responses were retained. Respondents included 96 males and 86 females.

\section{Measures}

To determine the relationship between interpersonal communication competence and exercise participation types, 16 items of exercise methods and 30 items of interpersonal communication competence measurement, excluding questionnaire about respondents, were used. There were 16 questions on exercise type, exercise goals, and exercise participation type; the questions also asked about the intent to join a club and use of apps. In addition, to measure interpersonal communication competence, an instrument developed by Rubin and Martin (1994) was translated and used. This instrument measures specific items in a total of 10 areas of interpersonal communication competence: self-disclosure, empathy, social relaxation, assertiveness, interaction management, altercentrism, expressiveness, supportiveness, immediacy, and environmental control. Here, self-disclosure refers to the behavior of disclosing personal traits to others through communication, and empathy refers to feeling one with others and is related to emotional responses. Social relaxation means having less anxiety in all social relationships and the ability to deal with negative responses or criticism from others without stress, and assertiveness is the ability to express one's own rights without denying the rights of others. Interaction management is the ability to handle all conversation procedures, including discussion topics, turn-taking, and the ability to negotiate the beginning and end of a conversation. Altercentrism is related to interest in others; it refers to paying attention to what others say and how they talk and recognizing spoken as well as unspoken messages. Expressiveness refers to the ability to use not only verbally accurate words but also nonverbal language that can appropriately express one's emotion. Supportiveness is the ability to make both parties feel that they are communicating in an equal atmosphere. Immediacy involves showing an attitude of immediate availability for communication with others, and linguistically, it means "immediate answers" or "focus on others." Environmental control is proving the ability to achieve prescribed goals, meet needs, deal with the negotiation environment, and solve problems.

The interpersonal communication competence questionnaire that measures these skills is composed of 10 subdimensions on a Likert scale, and total scores are used to compare interpersonal communication competence.

\section{Statistical analysis}

To compare communication competence and exercise participation types, the entry, processing, and statistical tests of the collected survey data were conducted using IBM SPSS Statistics ver. 25.0 (IBM Co., Armonk, NY, USA). The reliabilities of the questionnaire items measured by Cronbach alpha were 0.668 and 0.678 for exercise methods and interpersonal communication competence, respectively.

In addition, correlation and cross-tabulation analyses were conducted to determine the relationship between exercise participation types and communication competence, and independent $t$-tests and analysis of variance were carried out to compare the averages of the total scores of communication competence. In addition, logistic regression analysis was performed to determine the skills of communication competence that have significant influ- 
ence on exercise participation types.

\section{RESULTS}

\section{Exercise trends of respondents}

The exercise trends of respondents were identified to determine the relationship between communication competence and exercise participation types. Respondents' average numbers for exercise sessions per week and month were 2.45 and 9.05 , respectively. In terms of exercise type, personal exercise such as gym exercise was the most prevalent with $66.1 \%$, followed by group exercises such as baseball, soccer, and basketball with $16.1 \%$, and small group exercises such as badminton, golf, and tennis with $4.4 \%$. Most respondents (55.5\%) indicated that the goal for regular exercise was healthy living, which was followed by weight loss (21.4\%). Body shape correction $(2.7 \%)$ and exercise rehabilitation $(2.2 \%)$ had low numbers of responses. In terms of exercise participation types, respondents' answers to joining a club and using a fitness app are shown in Table 1.

Even though the current club join rate was only $20.3 \%$, a positive result, 3.28 out of 5 points, of future intent to join a club for exercise was obtained. Current use rate of fitness apps was low, at $22.0 \%$.

The most prevalent exercise participated in by the respondents who were club members was group exercise $(56.8 \%)$, followed by personal exercise $(27.0 \%)$ and small-group exercise $(5.4 \%)$. The correlation between exercise type and club membership was significant $(P<0.005)$. The correlation between app use and exercise type was also significant $(P<0.005)$, and app use was most frequent for personal exercise (85.0\%). This was followed by group exercise $(7.5 \%)$ and small-group exercise $(2.5 \%)$.

\section{Relationship between communication competence and club membership}

To determine the relationship between communication competence and sports club membership, club type, reason for joining, and influence on exercise adherence were investigated. First, average club membership was 18.7 months, and average duration of exercise per club visit was $2.5 \mathrm{hr}$. In terms of club type, the rate of offline membership (83.8\%) was significantly higher than online

Table 1. Types of exercise participation

\begin{tabular}{lccc}
\hline Type & Current joining rate & Mean future intent to join & Current use rate \\
\hline Clubs & $20.30 \%$ & 3.28 (out of 5 points) & - \\
Application use & - & - & $22.00 \%$ \\
\hline
\end{tabular}

membership (5.4\%) or both online and offline memberships $(10.8 \%)$. The most prevalent reason for joining a club was that the club was essential owing to the nature of the exercise (35.1\%), followed by greater effects of exercise when exercising with others (21.6\%), formation of various human relationships through clubs (18.9\%), and being with close friends or family (16.2\%). The average score for the question of whether a club is helpful in exercise adherence was 4.24 out of 5 points.

Next, the relationship with the communication competence of the respondents who exercise by joining a club was determined. First, the influence of the skills of communication competence of respondents who currently have a club membership was determined. The effect of joining a club in comparison to not joining a club was determined by performing logistic regression analysis, and it was found that only self-disclosure was significant, as shown in Table 2.

Next, a logistic regression analysis was performed to determine which skills of communication competence of the respondents who intend to join a club are more related in comparison to the respondents who do not intend to join a club. The results showed that social relaxation has a significant influence, as shown in Table 3.

\section{Relationship between communication competence and fitness app use}

To determine the relationship between communication competence and fitness app use, whether a fitness app is used, type of app used, reason for use, and the goal of exercise were investigated first. The results showed that the most prevalent apps were personal exercise management apps such as home training (50.8\%),

Table 2. Regression analysis of club membership and communication competence

\begin{tabular}{lccccc}
\hline Variable & $\mathrm{B}$ & $\mathrm{SE}$ & Wald & P-value & Exp (B) \\
\hline Self-disclosure & 0.259 & 0.133 & 3.770 & 0.052 & 1.295 \\
Empathy & -0.278 & 0.165 & 2.846 & 0.092 & 0.757 \\
Social relaxation & 0.063 & 0.136 & 0.211 & 0.646 & 1.065 \\
Assertiveness & 0.150 & 0.143 & 1.106 & 0.293 & 1.162 \\
Altercentrism & 0.227 & 0.147 & 2.401 & 0.121 & 1.255 \\
Interaction management & -0.163 & 0.143 & 1.299 & 0.254 & 0.850 \\
Expressiveness & -0.120 & 0.161 & 0.558 & 0.455 & 0.887 \\
Supportiveness & 0.133 & 0.146 & 0.831 & 0.362 & 1.143 \\
Immediacy & -0.204 & 0.154 & 1.754 & 0.185 & 0.816 \\
Environmental control & 0.308 & 0.175 & 3.101 & 0.078 & 1.360 \\
Constant & -5.253 & 1.930 & 7.040 & 0.007 & 0.005
\end{tabular}

B, coefficient for the constant in the null model; SE, standard error; Wald, Wald chisquare test that tests the null hypothesis that the constant equals 0 ; $\operatorname{Exp}(B)$, exponentiation of the $B$ coefficient. 
Table 3. Regression analysis of intention to join a club and communication competence

\begin{tabular}{lccccc}
\hline Variable & $\mathrm{B}$ & $\mathrm{SE}$ & Wald & $P$-value & Exp (B) \\
\hline Self-disclosure & -0.080 & 0.128 & 0.391 & 0.532 & 0.923 \\
Empathy & 0.000 & 0.152 & 0.000 & 1.000 & 1.000 \\
Social relaxation & 0.356 & 0.125 & 8.090 & 0.004 & 1.427 \\
Assertiveness & 0.104 & 0.135 & 0.586 & 0.444 & 1.109 \\
Altercentrism & 0.195 & 0.135 & 2.080 & 0.149 & 1.216 \\
Interaction management & -0.184 & 0.135 & 1.861 & 0.172 & 0.832 \\
Expressiveness & -0.148 & 0.144 & 1.053 & 0.305 & 0.862 \\
Supportiveness & -0.045 & 0.142 & 0.100 & 0.752 & 0.956 \\
Environmental control & 0.004 & 0.153 & 0.001 & 0.978 & 1.004 \\
Constant & -2.134 & 1.835 & 1.353 & 0.245 & 0.118 \\
\hline
\end{tabular}

$B$, coefficient for the constant in the null model; SE, standard error; Wald, Wald chisquare test that tests the null hypothesis that the constant equals 0; Exp (B), exponentiation of the $B$ coefficient.

followed by personal condition monitoring apps such as simple heart rate monitoring (14.8\%), management apps for managing diet and exercise schedules (13.1\%), and information-providing apps that improve existing exercise methods (13.1\%). The most prevalent response (59.6\%) for using fitness apps was that exercise information or personal health conditions can be easily checked. The most prevalent goal of exercise was for healthy living (49.2\%), followed by weight loss (32.8\%), body shape correction $(13.1 \%)$, and exercise rehabilitation (4.1\%).

To determine the relationship between the use of fitness apps and communication competence skills, a logistic regression analysis was performed. The results showed that immediacy among communication skills was significantly related, as seen in Table 4.

\section{DISCUSSION}

This study determined the relationship between communication competence, club membership, and fitness app use, and identified the subfactors of communication competence that affect exercise participation types (clubs, apps) to identify the relationship between communication competence and exercise participation types.

First, the reason for determining the relationship between exercise club membership and communication competence was that communication competence was expected to influence club membership because those activities are voluntary, and clubs are organized for interpersonal relationships among club members.

The results of a logistic regression analysis to determine the specific relationship between the two showed that self-disclosure in respondents who are currently club members and social relax-
Table 4. Regression analysis of fitness app use and communication competence

\begin{tabular}{lccccc}
\hline Variable & $\mathrm{B}$ & $\mathrm{SE}$ & Wald & P-value & Exp (B) \\
\hline Self-disclosure & 0.041 & 0.128 & 0.104 & 0.747 & 1.042 \\
Empathy & 0.122 & 0.163 & 0.561 & 0.454 & 1.130 \\
Social relaxation & -0.138 & 0.123 & 1.255 & 0.263 & 0.871 \\
Assertiveness & 0.224 & 0.141 & 2.535 & 0.111 & 1.251 \\
Altercentrism & -0.104 & 0.140 & 0.552 & 0.457 & 0.901 \\
Interaction management & 0.171 & 0.150 & 1.304 & 0.253 & 1.187 \\
Expressiveness & -0.042 & 0.156 & 0.072 & 0.789 & 0.959 \\
Supportiveness & -0.204 & 0.157 & 1.675 & 0.196 & 0.816 \\
Immediacy & 0.525 & 0.169 & 9.611 & 0.002 & 1.691 \\
Environmental control & -0.083 & 0.174 & 0.227 & 0.634 & 0.921 \\
Constant & -7.350 & 2.104 & 12.198 & 0.000 & 0.001 \\
\hline
\end{tabular}

$\mathrm{B}$, coefficient for the constant in the null model; SE, standard error; Wald, Wald chisquare test that tests the null hypothesis that the constant equals 0 ; $\operatorname{Exp}(B)$, exponentiation of the $B$ coefficient.

ation in respondents who intend to join a club were determined to be important influencing variables. The fact that an increase in self-disclosure ability increases the possibility (Table 2) for respondents to eventually join a club makes it possible to understand that the respondents who exercise as club members have a high level of mutual intimacy ability, and this is recognized as an important ability. Because self-disclosure is based on reciprocity of relationships rather than just opening up, it reflects the comprehensive recognition of relationships and exercise. Overall, however, the high relationship in terms of social relaxation (Table 3) and positive responses to club membership indicates that people who have a stable ability to manage relationships with others can be considered to be likely to participate in clubs since the same concerns have to be managed with unfamiliar people owing to the nature of clubs.

Next, the differences in the total scores of communication competence according to fitness app use were found using the $t$-test to determine the influence of communication competence skills on fitness app use. The score for fitness app users was significantly higher $(P<0.010)$ by 6.02 points, at 114.58 points, while that of nonusers was 108.56 points. In addition, the results of a logistic regression analysis performed to determine which skills of communication competence were related to fitness app use showed that immediacy was the important influencing variable (Table 4). According to Rubin and Martin (1994), immediacy refers to behavior that verbally and nonverbally reduces the psychological distance between people. Especially in verbal aspect, it means directly answering questions and focusing on the other person, and in that respect, it appears to attach great importance to immediate 
answers and the information necessary for exercise using fitness apps. This can be confirmed by the response that received the highest response frequency (15.4\%) to the question regarding why they use fitness apps: "because exercise information or personal health conditions can be easily checked." As such, the relationship between exercise participation types (clubs, fitness apps) and communication competence skills could be different in both types as well. This is due to the morphological characteristics of online and offline memberships and the number of participants between groups and individuals. Accordingly, the results of the regression analysis showed that social relaxation for respondents who exercise in a sports club and immediacy for the respondents who use fitness apps were significant, as shown in Tables 3 and 4 . Such differences can be considered in terms of the study by $\mathrm{Yi}_{1}$ and Kim (2018) that compared communication styles. The study investigated specifically which ability is related to style, which is a method of composing messages to others using the studied of Norton and Pettegrew (1977) and Norton (1978) on communication styles. Looking at immediacy and social relaxation based on the study of Yi and Kim (2018), their scores tend to be two extreme scores for the same style. For friendly style, attentive style, and precise style, immediacy had the highest or the second highest score, while social relaxation had the lowest score. On the other hand, both types had the highest scores for open style. Both apps and clubs have the characteristics of exposing private information to others, but apps have attentive and precise characteristics that confirm information accuracy and individually manage information. The insignificant relationship between friendliness and social relaxation can be understood by the report of $Y_{i}$ and Kim (2018), which stated that the communication stress of intimacy can hinder relaxation. This is also a reason for avoiding clubs. An interesting finding in the study of $Y_{1}$ and Kim (2018) is that the results of self-disclosure and social relaxation showed the same pattern, and both abilities showed low scores for styles for which immediacy had high scores. This appears to be related to the significant results of self-disclosure in respondents who are currently club members and social relaxation in respondents who intend to join a club in the future. Such a study is meaningful because it can be important data for exercise participants in selecting a type of exercise participation. The limitation of this study was, however, that it did not consider various types of participation because it focused on communication competence only. Contributions should be made to increase exercise adherence of exercise participants by comprehensively considering not only the diverse characteristics of exercise participants but also the characteristics of exercise participation types.

\section{CONFLICT OF INTEREST}

No potential conflict of interest relevant to this article was reported.

\section{ACKNOWLEDGMENTS}

This work was supported by the Ministry of Education of the Republic of Korea and the National Research Foundation of Korea (NRF-2017S1A6A3A01078538).

\section{REFERENCES}

Brawley LR, Carron AV, Widmeyer WN. Assessing the cohesion of teams: validity of the group environment questionnaire. J Sport Psychol 1987; 9:275-294.

Conroy DE, Yang CH, Maher JP. Behavior change techniques in top-ranked mobile apps for physical activity. Am J Prev Med 2014;46:649-652.

Curtis J, McTeer W, White P. Exploring effects of school experiences on sport participation in later life. Sociol Sport J 1999;16:348-365.

Gay V, Leijdekkers P. Personalised mobile health and fitness apps: lessons learned from myFitnessCompanion ${ }^{\circledR}$. Stud Health Technol Inform 2012;177:248-253.

Kim HS, Lee SA. An analysis in satisfaction rate of participation and sports activity continuation of the sports club participation. Korea J Sport Sci 2008;17:123-133.

Monge PR, Bachman SG, Dillard JP, Eisenberg EM. Communicator competence in the workplace: model testing and scale development. Ann Int Commun Assoc 1981;5:505-527.

Norton RW. Foundation of a communicator style construct. Human Com Res 1978;4:99-112.

Norton RW, Pettegrew L. Communicator style as an effect determinant of attraction. Com Res 1977;4:257-282.

Rubin RB, Martin MM. Development of a measure of interpersonal communication competence. Commun Res Rep 1994;11:33-44.

Spitzberg BH, Cupach WR. Handbook of interpersonal communication research. New York: Springer-Verlag; 1989.

Yi Y, Kim Y. Communication style perceived by Korean college students in their twenties-compared with communication competence. Cult Converg 2018;40:709-740.

Yuan S, Ma W, Kanthawala S, Peng W. Keep using my health apps: discover users' perception of health and fitness apps with the UTAUT2 model. Telemed J E Health 2015;21:735-741. 